\title{
Systematic Analysis of the ArcCheck Diode Arrays for Tomotherapy Delivery Verification
}

\author{
Qi Yue', Jimei Duan'1, Rongqing Li'1 ${ }^{1}$ Jack Yang² \\ ${ }^{1}$ Department of Radiation Oncology, First Affiliated Hospital of Kunming Medical University, Kunming, China \\ ${ }^{2}$ Department of Radiation Oncology, Monmouth Medical Center, Long Branch, NJ, USA \\ Email: jyang@barnabashealth.org
}

Received 12 August 2014; revised 10 September 2014; accepted 5 October 2014

Copyright (C) 2014 by authors and Scientific Research Publishing Inc.

This work is licensed under the Creative Commons Attribution International License (CC BY). http://creativecommons.org/licenses/by/4.0/

(c) (i) Open Access

\begin{abstract}
Objective: The aim of this study was to evaluate the functional characteristics of ArcCheck ${ }^{\mathrm{TM}}$ array detectors and the application for Tomotherapy IMRT plan verification. Method and Materials: The basic character of the ArcCheck applying on Tomotherapy system was tested. The diode testing procedures included the followings: The short term reproducibility, linearity, dose rate dependence, the profile measurement compared to ion chamber. The effect of insert on gamma passing rate was also studied. A total number of 602 Tomotherapy Delivery Quality Assurance (DQA) plans were retrospectively analyzed to obtain the action limit results with statistical significance evaluation. Results: The short term reproducibility, linearity, dose rate dependence, profile to ion chamber correlation and clinical application were all compared with satisfactory outcome with this device. If anterior diode points were properly calibrated with machine absolute output, then there was no significant difference between the ArcCheck phantom with and without insert on gamma passing rate. The average passing rate was $97.5 \%$ and the recommend action level was established at $92 \%$ based on the 602 patients' clinical data with $95 \%$ confidence level criteria. Conclusion: This comprehensive study shows that ArcCheck is an accurate and efficient device for quality assurance of Tomotherapy with remarkable consistency of 3D volume analysis of arc therapy.
\end{abstract}

\section{Keywords}

ArcCheck, Gamma Passing Rate, Tomotherapy

\section{Introduction}

Tomotherapy delivers IMRT using a rotating linear accelerator mounted on a moving slip ring gantry in con-

How to cite this paper: Yue, Q. Duan, J.M., Li, R.Q. and Yang, J. (2014) Systematic Analysis of the Arc Check Diode Arrays for Tomotherapy Delivery Verification. International Journal of Medical Physics, Clinical Engineering and Radiation Oncology, 3, 218-225. http://dx.doi.org/10.4236/ijmpcero.2014.34028 
junction with a binary multileaf collimator device. A 6MV photon beam radiation is continuously engaged while the couch is moving into the gantry continuously with a helical pattern [1]. Tomotherapy is much more complex compared to the conventional step and shoot Linac IMRT modality due to the helical delivery pattern which may have potential mismatch of gantry speed and couch motion for delivering errors. Quality assurance for Tomotherapy is important to assure the patients' treatment safety via utilizing a rotational style of phantom testing [2]-[4].

Films with Cheese phantom is a default methodology of Tomotherapy QA verification widely utilized to validate the planar dose distributions with overlaying phantom plans [5] [6]. However, the film QA process is time consuming and can be easily the calibration curve which may introduce dosimetry uncertainty [7]-[9]. Radiochromic film dosimetry was also widely implemented for IMRT QA, due to its simplicity and no need for chemical processors [10]-[11]. The calibration protocol has to be established to quantify the plannar dose distribution, with Then using the $2 \mathrm{D}$ detector arrays, such as Map Check ${ }^{\mathrm{TM}}$ and Matri $\mathrm{XX}^{\mathrm{TM}}$, they could easily acquire the dose map immediately after the irradiation, provide much more convenient data acquisition than the film and has been received wide acceptance in IMRT QA [12]-[14]. However, the geometry settings of planar dosimetry measurement to correlate to the rotational beam delivery have been challenged with dissimilar delivery techniques (rotational versus fixed beam angles). Devices with 2D arrays also have been successfully used for Tomotherapy DQA in early adaption [15] [16]. Similar questions have been raised that the 2D arrays have some limitation for the Tomotherapy DQA, due to the 2D array device has directional dependence which impaired the final dosimetry analysis with beam geometry correction [12]-[14]. For instance, a correction factor has to be applied when 2D array is reduced to 1D plane when the array is irradiated at 90 degrees, which the beam has penetrated to the series of diodes in the same plane. This limitation also causes some dosimetric errors in rotational DQA [15], experimental geometry correction factor needs to be implemented to obtain reasonable passing rate for Tomotherapy plans with rotational delivery.

The ArcCheck device (Sun Nuclear Corp., Melbourne, FL) which is a cylindrical acrylic phantom, has been developed for QA verification of Volumetric Modulated Arc Therapy (VMAT) delivery [17]-[19]. One of the major physical characteristics of ArcCheck is that from the Beams Eye View (BEV) projection, this device is consistent regardless of gantry angle of projections; therefore, those cylindrical detectors were designed to reduce the directional dependence [19]. The physical shape of ArcCheck made itself suitable for rotational DQA purpose. The aim of this study is to evaluate the detector characteristics of ArcCheck and also to apply the ArcCheck dosimetric principles for Tomotherapy verification.

\section{Material and Methods}

Helical Tomotherapy System, Planning Station 4. 0.4 DQA Station as well as the commercially available ArcCheck system were utilized in this study. Regarding the design features of ArcCheck, it is combination of detector arrays of 1386 diodes which are positioned over the surface of a cylinder tube, measuring $21 \mathrm{~cm}$ in radius and forming a spiral path of $1 \mathrm{~cm}$ separation and $1 \mathrm{~cm}$ distance from each diode detector. The buildup region consists water-equivalent phantom, with centrally customized adapter to fit the $0.056 \mathrm{~cm}^{3} \mathrm{~A} 1 \mathrm{SLion}$ chamber and connected with Tomo Electronmeter (Both manufactured by Standard Imaging, Middleton, WI, USA).

\subsection{Short Term Reproducibility}

All measurements were executed and data acquired with ArcCheck setup as fixed Source-to-Axis Distance (SAD) technique. The field size was $5 \mathrm{~cm} \times 20 \mathrm{~cm}$ (5 cm Jaw width, with central 32 MLC banks open) and the beam on time is set at 20 second. The ArcCheck was repeatedly irradiated for 10 times for statistical analysis. The ion chamber at the ArcCheck center was used to monitor the machine output changes. In order to accomplish the statistically meaningful output comparison, the ratio of the ion chamber reading to the average calibrated value of the two centrally located ArcCheck diodes was utilized to trace and correct the variation with machine output drifting. This process was repeated for 10 times, to avoid the statistical bias, the results were all normalized to the averaging acquired in the 10 trials. Therefore, the standard deviation (SD) with the 10 consecutive measurements was recorded and evaluated to assess the ArcCheck reproducibility with the same setups.

\subsection{Linearity}

The measurement was acquired at a SAD method with same filed size defined in the previous section. The dose 
linearity response of the ArcCheck was evaluated by measuring the data of the two central diodes on an average with beam on time intervals varying from $5 \mathrm{~s}$ to $60 \mathrm{~s}$, respectively.

\subsection{Dose per Pulse Dependence}

The dose rate dependence was measured by changing the ArcCheck phantom setup Source-to-Detector Distance (SDD) from $65 \mathrm{~cm}$ to $85 \mathrm{~cm}$ on Tomotherapy with a fixed field size of $5 \mathrm{~cm} \times 20 \mathrm{~cm}$ and $20 \mathrm{~s}$ beam on time. The reading at each SDD was normalized to the reading at $85 \mathrm{~cm}$ SDD. The results were compared to the ion chamber measured at the same irradiation conditions, which serves as the standard for variation analysis.

\subsection{Longitude Profile Comparison}

The measurement technique was chosen on Tomotherapy with 5 Jaw width and all leaf open at 85 SDD setup, beam on time is also set at $20 \mathrm{~s}$. Then the longitude profile was obtained by collecting data from the ArcCheck central axis diode with individual setting. The ArcCheck profile was then compared with the profile obtained by topography scan with the ion chamber inserted in solid water equivalent phantom under the same irradiation condition. Those profiles were also compared to check the corresponding performance with beam profiles.

\subsection{Gamma Passing Rates for ArcCheck with Acrylic Insert and without Acrylic Insert}

The ArcCheck was scanned on Tomotherapy MVCT with insert and without insert with separate CT images. The images of ArcCheck with insert and without insert were sent to Tomotherapy DQA station as DQA phantom. Ten deliverable plans were randomly selected to apply the DQA based on two serial ArcCheck images with and without insert. The Gamma passing rate of ArcCheck with insert were compared to the passing rate of ArcCheck without insert for each plan, the absolute and relative dose outputs were analyzed.

\subsection{Clinical Application}

With those 602 HT plans, which were performed pretreatment verification on the Tomotherapy in the past three years, were retrospectively analyzed. The gamma method was then used to quantitatively compare the dose measured by ArcCheck and the dose calculated by planning station [20]. According the TG-119 report, the particular set was chosen: 3\% dose difference, $3 \mathrm{~mm}$ Distance to Agreement (DTA), Van Dyk criteria of 10\% threshold, and absolute dose comparison [19]. The treatment plans were re-grouped according the patient's disease sites: Brain, H \& N, Breast/Chest, Lung/esophagus, Abdomen, Pelvis, and other disease sites.

The Clinical action levels were established as the TG-119 reports' suggestion: For a large number of gamma analyses, $95 \%$ of the tests should result in pass rates that exceed $(100-C L) \%$, which the confidence limit is defined as $\mathrm{CL}=(100-$ mean $)+1.96 \sigma \quad[21]$.

\section{Results}

The rotational nature of Tomotherapy IMRT created multiple digress of freedom in treatment delivery for highly modulated fluence maps, however, the quality assurance also presented great challenges to daily operation due to the complexity of QA procedures of 3D data analysis. According to the ACR clinical practice guidelines, patient specific QA was still required to prove the clinical efficacy of VMAT treatment quality. Therefore, we have retrospectively collected the planning QA data to study the ArcCheck system responses with the following results with 602 patients' data.

Short term reproducibility: The short term reproducibility of the ArcCheck on Tomotherapy was found with a SD of $0.064 \%$ among the output drifting within $\pm 1 \%$ ranges in 10 continuous measurements. The output of data with the 10 measurements was shown in Figure 1.

Dose linearity: Measurement data indicated that the dose linearity ranges from $5 \mathrm{~s}$ to $60 \mathrm{~s}$ presented a straight function line, with the $\mathrm{R}^{2}$ value better than 0.999 (close to linear slope). Tomotherapy output functions along with ArcCheck measurements presented linear responses over a range of $5 \mathrm{~s}$ to $60 \mathrm{~s}$. With beam on time increments from $5 \mathrm{~s}$ to $60 \mathrm{~s}$, the measured dose relationship was shown in Figure 2.

Dose per pulse dependence: The dose function with various sources to detector distance could be a deterministic factor for planning dose distribution. Theoretically, it should follow the inverse square law correction, and 


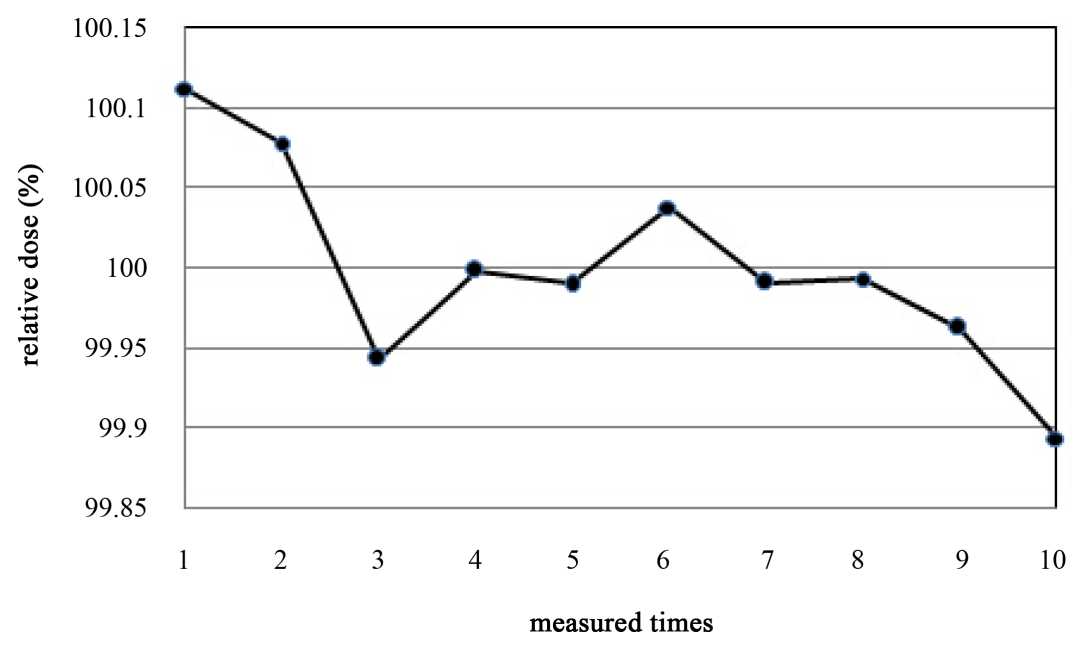

Figure 1. Short term reproducibility.

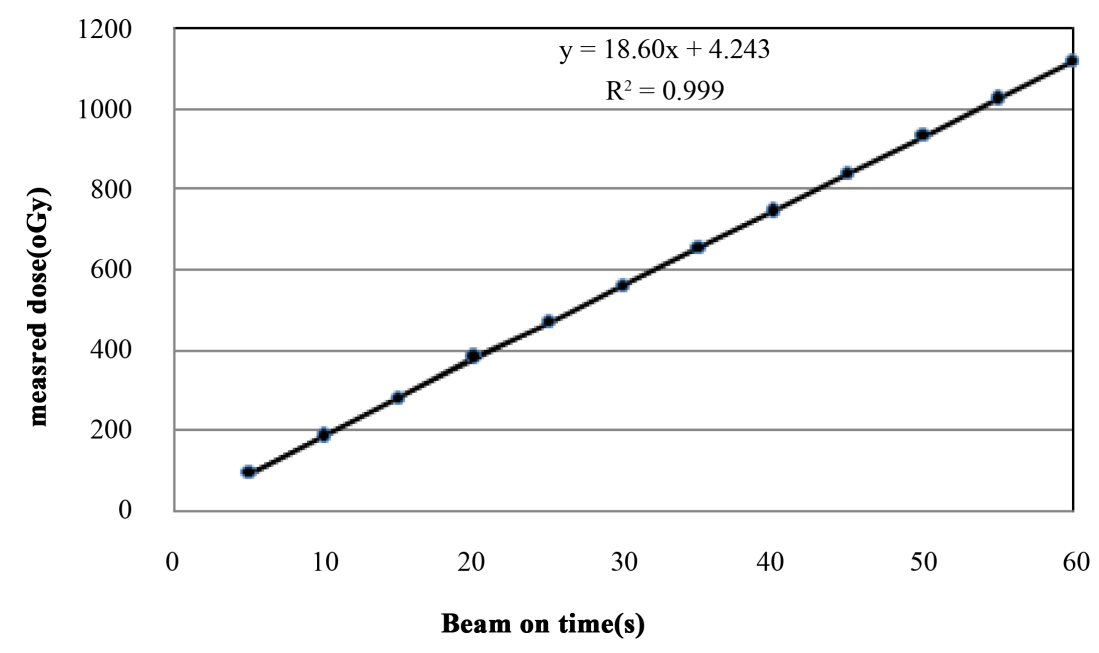

Figure 2. The response of linearity of dose and beam on time.

without detector dependency. Since the detector plans of ArcCheck were at the peripheral area, therefore, the response function had to be addressed. The dose rate dependence of the ArcCheck for SDD ranging from $65 \mathrm{~cm}$ to $85 \mathrm{~cm}$ was shown in Figure 3. By comparing with ArcCheck and ion chamber distance correlation, the response of the ArcCheck was 2.06\% higher than ionization chamber at the SDD of $65 \mathrm{~cm}$, which presented the diode over-response characteristics with closer SSD measurement.

Longitude profile comparison: The longitude profile measured by ArcCheck has been compared with ionization chamber responses. The result was shown in Figure 4. All measured data were normalized to the central axis. The $5 \mathrm{~cm}$ Jaw width profile comparison showed good agreement with ionization chamber.

ArcCheck with insert and without insert comparison: ArcCheck provided two different models for the VMAT measurements, which can be classified into a solid versus hollow settings. The major difference between these two delivery models counts on the mechanism with/without ion chamber measurements at the center of the cavity. Benefits for extra chamber measurements with plug usually supported benchmark or baseline with absolute output confirmation. However, if properly calibrated with the reference diode in the central beam, ArcCheck could still be utilized for absolute dosimetry without any ion chamber in the plug. In this study, we have acquired data for both settings to evaluate the gamma passing rate with $3 \% / 3 \mathrm{~mm}$ criteria. The gamma passing rate of DQA using ArcCheck with insert and without insert were 98.61\% $\pm 1.38 \%$ and $98.30 \% \pm 1.4 \%(p>0.05)$, respectively. The set passing rate were established under the $3 \% / 3 \mathrm{~mm}$ gamma criteria with this evaluation. The result was shown in Figure 5. 




Figure 3. The response curves of dose per pulse dependence.

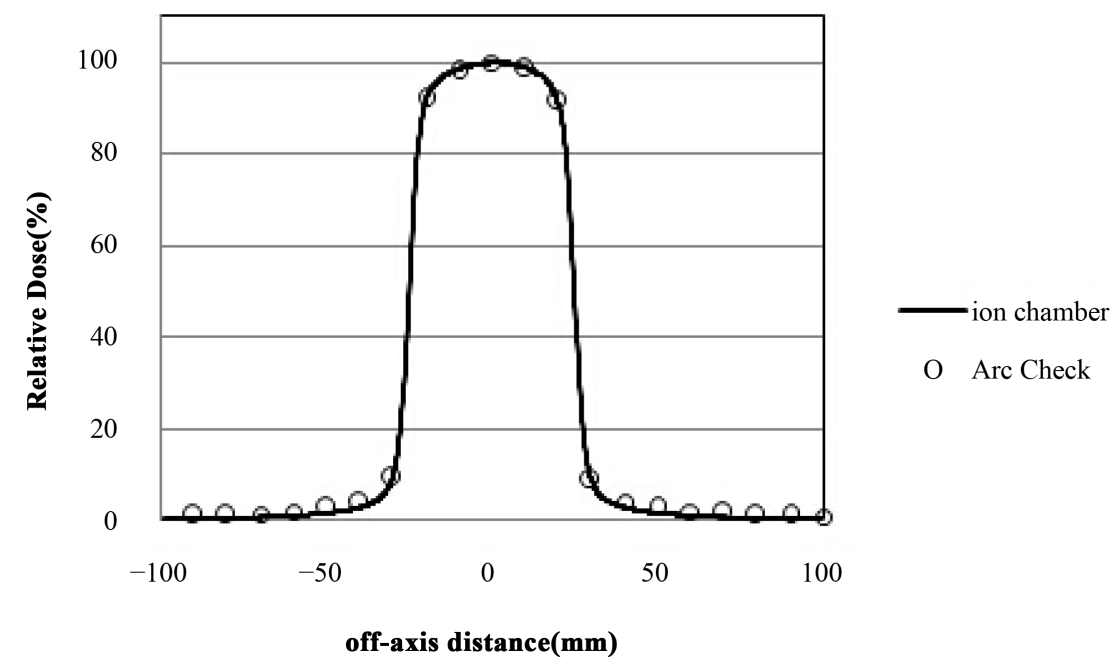

Figure 4. Longitude profile obtained by ArcCheck and ion chamber.

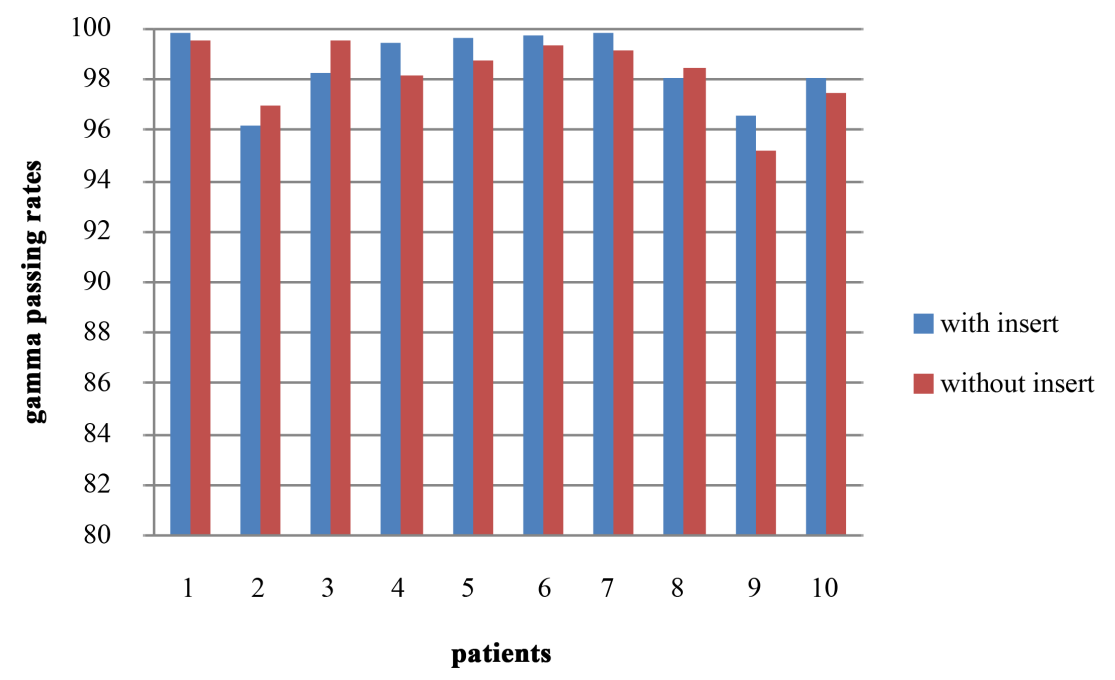

Figure 5. Gamma passing rate of ArcCheck with insert and without insert. 
Clinical implication: With total 602 clinical patients' verification plans, the gamma passing rates were summarized with various disease sites and then summarized in Table 1. According to the TG-119 reports and our departmental pre-set criteria for acceptable level of treatment verification, the suggested action level on our department is $92 \%$, based on the statistical analysis for all the cases we analyzed here.

\section{Discussion}

The ArcCheck device has been used for Tomotherapy DQA as well as Linac based VMAT patient specific QA. In the present study, the basic functional performance of the ArcCheck diode was tested on Tomotherapy system. Among our 602 deliverable plans, the short term reproducibility and linearity are relatively similar to the results obtained from the publication by Li et al. [17], which addressed the clinical issues with ArcCheck tested on Linac performance. In our results, the ArcCheck diodes demonstrated a good short-term output reproducibility and dose linearity, as expected and verified from the specification provided by vendor itself. Li et al. also reported the diode response exhibited a decreasing sensitivity variation of $1.4 \%$ and $0.6 \%$ with increasing SAD of 85 $125 \mathrm{~cm}$. By comparing with calculated and measured doses with previous data [17], the Tomotherapy system dose per pulse dependence of ArcCheck diode for SAD of 65 - $85 \mathrm{~cm}$ followed a similar function, with higher output for a closer SDD.

While the option of TomoDirect ${ }^{\mathrm{TM}}$ was not installed on our system, the angle dependence of the ArcCheck was not tested in our case study. Feygelman et al. and Li et al. both have reported the angler dependence of ArcCheck on Linac based systems [17] [18]; Li et al. also has reported that 14\% maximum variation was observed from $105^{\circ}$ to $180^{\circ}$ incident beam angle, giving an average of $0.19 \%$ response change per degree [17]. For the beam profile constancy evaluation, the longitudinal profile of $5 \mathrm{~cm}$ Jaw width obtained by ArcCheck and ion chamber were also tested on Tomotherapy system. The ArcCheck profile was in close agreement to the ion chamber topo scan data, with the tolerance less than $1 \mathrm{~mm}$ of Full Width Half Maximum (FWHM).

Examining the specification, the mid portion of ArcCheck device has a $15 \mathrm{~cm}$ diameter central cavity where could be filled with suitable dosimetric insert. H. Fair et al. reported development of a nonhomogeneous ArcCheck insert for DQA of VMAT [22]. The author designed and customized heterogeneous insert with densities to simulate different human tissues. The ArcCheck could also be measured without insert, by imaging this device differently for calculation. In this paper, the comparison between the phantom with homogeneous insert and without insert was tested thoroughly via gamma passing rate criteria. The results showed that there was no significant difference between the acquired data with insert and without insert on gamma passing rate.

The gamma method was commonly used to quantitatively compare measured and calculated dose map in order to determine the quality factor of an IMRT plan delivery. The gamma index has been widely investigated to establish the action level for patient QA. According the summary from TG-119 report, 95\% of such measurements should fall within the confidence level of one standard deviation [19]. Li et al. reported the DQA passing rate action levels for Tomotherapy and Linac based IMRT using Map Check ${ }^{\mathrm{TM}}$ were 87\% and 90\%, respectively [15]. Kim et al. suggested tolerance levels for Linac based IMRT DQA measurements using confidence limits

Table 1. Gamma passing rates of 602 in different disease sites.

\begin{tabular}{|c|c|c|c|}
\hline Sites & Number of Study & Mean \pm SD (\%) & Range (\%) \\
\hline Brain & 111 & $97.95 \pm 2.08$ & $90.3-100$ \\
\hline $\mathrm{H} \& \mathrm{~N}$ & 153 & $97.28 \pm 2.88$ & $82.9-100$ \\
\hline Chest/Breast & 20 & $95.61 \pm 4.25$ & $84.9-99.7$ \\
\hline Lung/Esophagus & 79 & $97.04 \pm 2.89$ & $85.0-100$ \\
\hline Abdomen & 48 & $98.07 \pm 1.92$ & $92.8-100$ \\
\hline Pelvis & 147 & $97.95 \pm 2.31$ & $90.2-100$ \\
\hline Bone & 24 & $96.68 \pm 3.10$ & $90.1-99.9$ \\
\hline Others & 20 & $96.59 \pm 3.48$ & $87.3-99.9$ \\
\hline Overall & 602 & $97.50 \pm 2.68$ & $82.9-100$ \\
\hline
\end{tabular}


determined by multi-institutional study was established on $87.9 \%$ at $3 \% / 3 \mathrm{~mm}$ [23]. Recent article proposed by Nelms et al. has raised questions about the gamma passing rate standard might not be sensitive enough to detect the IMRT delivery errors via clinical cases demonstration [24], we also were aware of those clinical issues which could be another area for further IMRT QA investigation. However, our study here simply just reviewed the physical functionalities of ArcCheck device for its utilization in routine Tomotherapy delivery quality evaluations. The statistical analysis of its stability associated with current gamma passing standards was the main purpose of this investigational work.

In this submission, we analyzed the gamma passing rates of 602 patients for Tomotherapy using the ArcCheck device. $98.2 \%$ of the plans had the gamma passing rate higher than $90 \% .84 .8 \%$ of the plans had the gamma passing rate higher than 95\% confidence level. Therefore, based on our own measurement data and the recommendation from TG-119 report to achieve 95\% measurements falling in one standard deviation confidence level, we then obtained the action level of our own department criteria of $92 \%$ passing rate with $3 \mathrm{~mm} / 3 \%$ setting for IMRT delivery quality review.

\section{Conclusion}

The ArcCheck device implemented clinically in our department apparently provided stable and consistent measurement outcomes with Tomotherapy helical therapy. The physical characteristics or ArcCheck and 602 clinical cases were summarized in details to show the simplicity and consistency in IMRT QA work. Tomotherapy, like other volumetric therapy delivery modalities, presented a very challenging situation for dose validation which directly influenced the IMRT qualities, both in planning and treatment delivery. Using the current acceptable methodology such as gamma passing rate evaluation, we have concluded that the ability of the ArcCheck device not only could measure helical Tomotherapy planning delivery, perform clinical dose measurements; but also could simplify as well as reduce the Tomotherapy QA workload in a very busy clinic (we are treating more than 40 patients per day on the Tomotherapy). This extensive study has shown that ArcCheck is a geometrically accurate and dosimetrically consistent device for quality assurance of Tomotherapy delivery. ArcCheck device provides good reducibility, is comparable to other commercialized systems used for similar volumetric treatment delivery validation QA.

\section{References}

[1] Mackie, T.R., Holmes, T., Swerdloff, S., et al. (1993) Tomotherapy: A New Concept for the Delivery of Conformal Radiotherapy. Medical Physics, 20, 1709-1719. http://dx.doi.org/10.1118/1.596958

[2] Fenwick, J.D., Tomé, W.A., Jaradat, H.A., et al. (2004) Quality Assurance of a Helical Tomotherapy Machine. Physics in Medicine and Biology, 49, 2933-2953. http://dx.doi.org/10.1118/1.596958

[3] Thomas, S.D., Mackenzie, M., Field, G.C., et al. (2005) Patient Specific Treatment Verifications for Helical Tomotherapy Treatment Plans. Medical Physics, 32, 3793-800. http://dx.doi.org/10.1118/1.2134929

[4] Langen, K.M., Papanikolaou, N., Balog, J., et al. (2010) QA for Helical Tomotherapy: Report of the AAPM Task GROUP 148. Medical Physics, 37, 4817-4853. http://dx.doi.org/10.1118/1.2134929

[5] Delivery Quality Assurance Guide, Accuray Corporation.

[6] Yan, Y., Papanikolaou, N., Weng, X., et al. (2005) Fast Radiographic Film Calibration Procedure for Helical Tomotherapy Intensity Modulated Radiation Therapy Dose Verification. Medical Physics, 32, 1566-1570. http://dx.doi.org/10.1118/1.2134929

[7] Zhu, X.R., Jursinic, P.A., Grimm, D.F., et al. (2002) Evaluation of Kodak EDR2 Film for Dose Verification of Intensity Modulated Radiation Therapy Delivered by a Static Multileaf Collimator. Medical Physics, 31, 2284-2288.

[8] Shi, C., Papanikolaou, N., Yan, Y., et al. (2006) Analysis of the Sources of Uncertainty for EDR2 Film-Based IMRT Quality Assurance. Journal of Applied Clinical Medical Physics, 25, 1-8.

[9] Buonamici, F.B., Compagnucci, A., Marrazzo, L., et al. (2012) An Intercomparison between Film Dosimetry and Diode Matrix for IMRT Quality Assurance. Medical Physics, 39, 6339-6350.

[10] Lewis, D., Micke, A., Yu, X. and Chan, M. (2014) An Efficient Protocol for Radiochromic Film Dosimetry Combining Calibration and Measurement in a Single Scan. Medical Physics, 13, 57-67.

[11] Nelms, B.E., Zhen, H. and Tomé, W.A. (2011) Per-Beam, Planar IMRT QA Passing Rates Do Not Predict Clinically Relevant Patient Dose Errors. Medical Physics, 38, 1037-1044. http://dx.doi.org/10.1118/1.3544657

[12] Jursinic, P.A. and Nelms, B.E. (2003) A 2-D Diode Array and Analysis Software for Verification of Intensity Mod- 
ulated Radiation Therapy Delivery. Medical Physics, 30, 870-879. http://dx.doi.org/10.1118/1.1567831

[13] Létourneau, D., Gulam, M., Yan, D., Oldham, M. and Wong, J.W. (2004) Evaluation of a 2D Diode Array for IMRT Quality Assurance. Radiology and Oncology, 70, 199-206. http://dx.doi.org/10.1016/j.radonc.2003.10.014

[14] Li, J.G., Yan, G. and Liu, C. (2009) Comparison of Two Commercial Detector Arrays for IMRT Quality Assurance. Journal of Applied Clinical Medical Physics, 10, 2942.

[15] Chong, N.S., Lee, J.J.S., Kung, W.-H., et al. (2011) Patient Delivery Quality Assurance for Linac-Based IMRT and Helical Tomotherapy Using Solid State Detectors. Radiation Measurements, 46, 1993-1995. http://dx.doi.org/10.1016/j.radmeas.2011.08.004

[16] Xu, S., Xie, C., Ju, Z., Dai, X., Gong, H., Wang, L. and Yang, J. (2010) Dose Verification of Helical Tomotherapy Intensity Modulated Radiation Therapy Planning Using 2D-Array Ion Chambers. Biomedical Imaging and Intervention Journal, 6, e24. http://dx.doi.org/10.2349/biij.6.2.e24

[17] Li, G., Zhang, Y., Jiang, X., Bai, S., Peng, G., Wu, K. and Jiang, Q.F. (2013) Evaluation of the ArcCHECK QA System for IMRT and VMAT Verification. Physica Medica: European Journal of Medical Physics, 29, 295-303. http://dx.doi.org/10.1016/j.ejmp.2012.04.005

[18] Feygelman, V., Zhang, G., Stevens, C. and Nelms, B.E. (2011) Evaluation of a New VMAT QA Device, or the "X” and “O” Array Geometries. Journal of Applied Clinical Medical Physics, 12, 3346.

[19] (2008) ArcCheck ${ }^{\mathrm{TM}}$ Reference Guide. Sun Nuclear Corporation.

[20] Depuydt, T., Van Esch, A. and Huyskens, D.P. (2002) A Quantitative Evaluation of IMRT Dose Distributions: Refinement and Clinical Assessment of the Gamma Evaluation. Radiotherapy and Oncology, 62, 309-319. http://dx.doi.org/10.1016/S0167-8140(01)00497-2

[21] Ezzel, G.A., Burmeister, J.W., Dogan, N., et al. (2009) IMRT Commissioning: Multiple, Institution Planning and Dosimetry Comparisons, a Report from AAPM Task Group 119. Medical Physics, 36, 5359-5373. http://dx.doi.org/10.1118/1.3238104

[22] Fakir, H., Gaede, S., Mulligan, M. and Chen, J.Z. (2012) Development of a Novel ArcCHECK Insert for Routine Quality Assurance of VMAT Delivery Including Dose Calculation with Inhomogeneities. Medical Physics, 39, 42034208. http://dx.doi.org/10.1118/1.4728222

[23] Kim, J.I., Chung, J.B., Park, Y.K., et al. (2013) A Multi-Institutional Study for Tolerance and Action Levels of IMRT Dose Quality Assurance Measurements in Korea. Journal of Applied Clinical Medical Physics, 14, 3964.

[24] Nelms, B.E., Chan, M.F., Jarry, G., Lemire, M., Lowden, J., Hampton, C. and Feygelman, V. (2013) Evaluating IMRT and VMAT Dose Accuracy: Practical Examples of Failure to Detect Systematic Errors When Applying a Commonly Used Metric and Action Levels. Medical Physics, 40, 111722. 
Scientific Research Publishing (SCIRP) is one of the largest Open Access journal publishers. It is currently publishing more than 200 open access, online, peer-reviewed journals covering a wide range of academic disciplines. SCIRP serves the worldwide academic communities and contributes to the progress and application of science with its publication.

Other selected journals from SCIRP are listed as below. Submit your manuscript to us via either submit@scirp.org or Online Submission Portal.
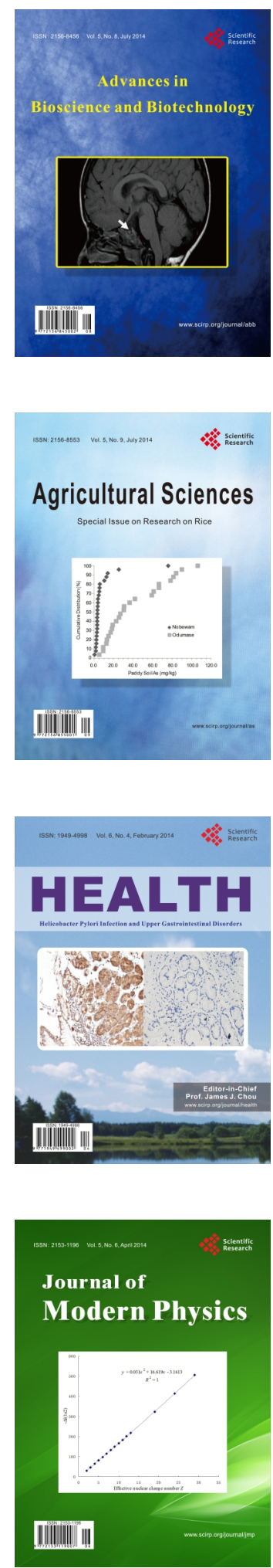
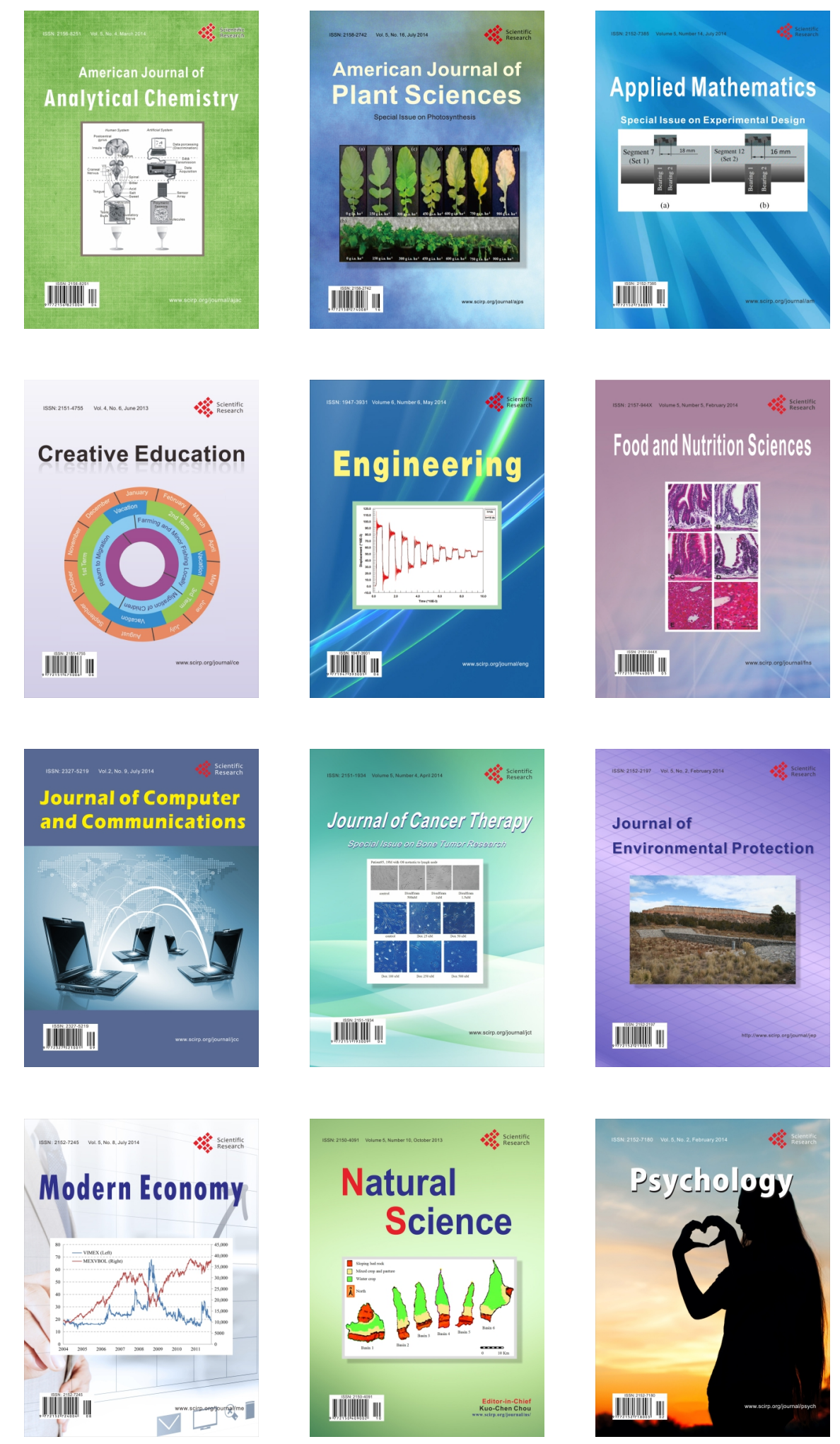